\title{
Almost Sure Exponential Stabilization by Discrete-time Stochastic Feedback Control
}

\author{
Xuerong Mao, Senior Member, IEEE
}

\begin{abstract}
Given an unstable linear scalar differential equation $\dot{x}(t)=\alpha x(t)(\alpha>0)$, we will show that the discrete-time stochastic feedback control $\sigma x([t / \tau] \tau) d B(t)$ can stabilize it. That is, we will show that the stochastically controlled system $d x(t)=\alpha x(t) d t+\sigma x([t / \tau] \tau) d B(t)$ is almost surely exponentially stable when $\sigma^{2}>2 \alpha$ and $\tau>0$ is sufficiently small, where $B(t)$ is a Brownian motion and $[t / \tau]$ is the integer part of $t / \tau$. We will also discuss the nonlinear stabilization problem by a discretetime stochastic feedback control. The reason why we consider the discrete-time stochastic feedback control is because that the state of the given system is in fact observed only at discrete times, say $0, \tau, 2 \tau, \cdots$, for example, where $\tau>0$ is the duration between two consecutive observations. Accordingly, the stochastic feedback control should be designed based on these discrete-time observations, namely the stochastic feedback control should be of the form $\sigma x([t / \tau] \tau) d B(t)$. From the point of control cost, it is cheaper if one only needs to observe the state less frequently. It is therefore useful to give a bound on $\tau$ from below as larger as better.
\end{abstract}

Index Terms-Brownian motion, stochastic differential delay equations, difference equations, almost sure exponential stability, discrete-time feedback control, stochastic stabilization.

\section{INTRODUCTION}

It is well known that noise can be used to stabilize a given unstable system or to make a system even more stable when it is already stable. For example, the linear scalar differential equation $\dot{x}(t)=\alpha x(t)$ is unstable when $\alpha>0$ but it can be stabilized by a Brownian motion. In fact, the linear stochastic differential equation (SDE)

$$
d x(t)=\alpha x(t) d t+\sigma x(t) d B(t)
$$

is almost surely exponentially stable if and only if $\sigma^{2}>2 \alpha$ (see e.g. [3], [12], [18], [25]). From the point of control theory, it is the stochastic feedback control $\sigma x(t) d B(t)$ that stabilizes the unstable system $\dot{x}(t)=\alpha x(t)$. The pioneering work in this area was due to Hasminskii [12], who stabilized a system by using two white noise sources. Later, Arnold et al. [4] showed that the multi-dimensional linear system $\dot{x}(t)=A x(t)$ can be stabilized by zero mean stationary parameter noise if and only if $\operatorname{trace}(A)<0$. In the nonlinear case, Scheutzow [26] provided us with some examples on stabilization and destabilization in the plane. Mao [17] developed a general theory on stabilization and destabilization by Brownian motion. In [17], Mao showed that given a multi-dimensional unstable nonlinear system $\dot{x}(t)=f(x(t), t)$ whose coefficient satisfies the linear growth condition $|f(x, t)| \leq K|x|$ ( $K$ is a positive constant), it is possible to design a linear stochastic feedback control

X. Mao is with the Department of Mathematics and Statistics, University of Strathclyde, Glasgow G1 1XH, UK. x.mao@strath.ac.uk
$A x(t) d B(t)$ based on a scalar Brownian motion $B(t)$ so that the stochastically controlled system

$$
d x(t)=f(x(t), t) d t+A x(t) d B(t)
$$

becomes almost surely exponentially stable. Appleby and Mao [2] generalized the results above in order to stabilize a class of functional differential equations by noise.

Before we introduce the discrete-time control problem, it is better to compare the above stochastic feedback control with the deterministic feedback control. Consider an unstable multi-dimensional linear system $\dot{x}(t)=A x(t)$. In the classical way, we could design a deterministic state feedback control so that the controlled system $\dot{y}(t)=A y(t)+C y(t)$ becomes stable. Here we change $x(t)$ into $y(t)$ in order to indicate more clearly that the states of the original and controlled systems are different. As a matter of fact, we know that $x(t) \neq$ $y(t)$. On the other hand, one could design a stochastic state feedback control so that the stochastically controlled system $d z(t)=A z(t) d t+C z(t) d B(t)$ becomes stable, where $B(t)$ is a Brownian motion. Such a stochastic state feedback control has its advantage: it preserves the original state in average, namely $\mathbb{E} z(t)=x(t)$. In other words, the provided stochastic feedback control method achieves sample-path stabilization, while the expectation of the state is, at all times, equal to the state of the original uncontrolled system. The stochastic state feedback control has been used in engineering (see e.g. [12], [22] and the references therein). We should also mention that this interesting phenomenon has also been observed in finance and ecosystem. A Nobel Prize winning model, namely the Black-Scholes model [5] for a share price is described by the scalar linear $\operatorname{SDE} d z(t)=\alpha z(t) d t+\sigma z(t) d B(t)$, where $\alpha$ is the growth rate and $\sigma$ the volatility. The overall (average) share price $x(t):=E z(t)$ satisfies the differential equation $\dot{x}(t)=\alpha x(t)$ so it will grow exponentially when the growth rate $\alpha>0$. However, if the volatility $\sigma$ is sufficiently large (namely $0.5 \sigma^{2}>\alpha$ ), the individual price $z(t)$ will tend to zero with probability one. This reveals an important phenomenon that although the overall market grows, an individual share holder might still lose a fortune in the large volatility situation. This concept has been developed in mathematical finance and is now known as the volatility-stabilized markets (see e.g. [9]). In ecosystem, the SDE models have revealed another important phenomenon that the environmental noise might make a population become extinct (see e.g. [18, Chapter 11]).

Let us now introduce the discrete-time stochastic feedback control. We observe that a common feature of the stochastic feedback controls discussed above is that the controls depend on the current state $x(t)$ continuously. For example, in the 
SDE (1.1), the stochastic feedback control $\sigma x(t) d B(t)$ requires the continuous observation of the state $x(t)$ for all time $t \geq 0$. However, in practice, the state is observed only at discrete times, say $0, \tau, 2 \tau, \cdots$, for example, where $\tau>0$ is the duration between two consecutive observations (see e.g. [8] and the references therein). It also costs less if $\tau$ is larger. Accordingly, the stochastic feedback control should be designed based on these discrete-time observations, namely the stochastic feedback control should be of the form $\sigma x([t / \tau] \tau) d B(t)$, where $[t / \tau]$ is the integer part of $t / \tau$. The problem is therefore to find out if the following stochastically controlled system

$$
d x(t)=\alpha x(t) d t+\sigma x([t / \tau] \tau) d B(t)
$$

is almost surely exponentially stable when $\sigma^{2}>2 \alpha$ ? One of our key aims in this paper is to give a positive answer to this problem. We will show that this controlled system is almost surely exponentially stable for sufficiently small $\tau$, namely $\tau<\tau^{*}$ and we will estimate $\tau^{*}$ from below.

It should be pointed out that the corresponding problem for the deterministic differential equations has been studied by many authors (see e.g. [1], [6], [7], [10], [11]). However, the almost surely stochastic stabilization problem (1.3) has not been studied so far. After we prove the stability of the linear system (1.3) in Section 2, we will generalize our result to the multi-dimensional non-linear case in Section 3. The reader may wonder if one could look at the multi-dimensional nonlinear case first and then, from this, obtain the result for the scalar linear case. The reason why we do not do so is because we would not be able to obtain good estimation on $\tau^{*}$, the lower bound for $\tau$ (see above), in the scalar linear case. In fact, as we will see below that the proof of the scalar linear case makes the full use of the explicit solution and the bound on $\tau^{*}$ is significantly better than nonlinear case. We do not have this advantage in the nonlinear case. We therefore have to develop a completely different approach to the nonlinear discrete-time controlled problem.

\section{SCALAR Linear EQUATIONS}

Throughout this paper, unless otherwise specified, we let $\left(\Omega, \mathcal{F},\left\{\mathcal{F}_{t}\right\}_{t \geq 0}, \mathbb{P}\right)$ be a complete probability space with a filtration $\left\{\mathcal{F}_{t}\right\}_{t \geq 0}$ satisfying the usual conditions (i.e. it is increasing and right continuous while $\mathcal{F}_{0}$ contains all $\mathbb{P}$-null sets). Let $B(t)$ be a scalar Brownian motion defined on the probability space.

Consider the scalar linear stochastic equation

$$
d x(t)=\alpha x(t) d t+\sigma x([t / \tau] \tau) d B(t)
$$

on $t \geq 0$ with initial value $x(0)=x_{0} \in \mathbb{R}$, where $\tau$ is a positive constant. Let us form this equation as a stochastic differential delay equation (SDDE). In fact, if we define a time-dependent delay function $\delta:[0, \infty) \rightarrow[0, \tau]$ by

$$
\delta(t)=t-k \tau \text { for } t \in[k \tau,(k+1) \tau), k=0,1,2, \cdots,
$$

then equation (2.1) can be re-written as the following SDDE

$$
d x(t)=\alpha x(t) d t+\sigma x(t-\delta(t)) d B(t) .
$$

It is therefore well known that equation (2.1) has a unique solution $x(t)$ on $t \geq 0$ and, moreover, any moment of the solution is finite (see e.g. [13], [14], [15], [16], [23]).

Theorem 2.1: If

$$
\alpha-\frac{\sigma^{2}}{2}<0
$$

then there is a positive number $\tau^{*}$ such that for any initial value $x_{0} \in \mathbb{R}$, the solution of equation (2.1) satisfies

$$
\limsup _{t \rightarrow \infty} \frac{1}{t} \log (|x(t)|)<0 \quad \text { a.s. }
$$

provided $\tau \in\left(0, \tau^{*}\right)$. In practice, we can choose a positive number $p \in(0,1)$ for which

$$
\alpha-\frac{(1-p) \sigma^{2}}{2}<0
$$

and let $\tau^{*}$ be the smallest positive root to the equation

$$
H_{1}(\tau)+H_{2}(\tau)=0,
$$

where

$$
H_{1}(\tau)=p\left(e^{\alpha \tau}-1\right)+\frac{p(p-1) \sigma^{2}}{4 \alpha}\left(e^{2 \alpha \tau}-1\right)
$$

and

$$
\begin{aligned}
& H_{2}(\tau)=\frac{p(p-1)}{2}\left(e^{\alpha \tau}-1\right)^{2} \\
& +\frac{p(p-1)(p-2)}{6}\left[\left(e^{\alpha \tau}-1\right)^{3}+3\left(e^{\alpha \tau}-1\right) \hat{\sigma}^{2}\right] \\
& +\frac{p(p-2)(2 p-7)}{8}\left[\left(e^{\alpha \tau}-1\right)^{4}+6\left(e^{\alpha \tau}-1\right)^{2} \hat{\sigma}^{2}+3 \hat{\sigma}^{4}\right] \\
& +\frac{p(p-2)(p-4)}{8}\left[\left(e^{\alpha \tau}-1\right)^{5}+10\left(e^{\alpha \tau}-1\right)^{3} \hat{\sigma}^{2}\right. \\
& \left.+15\left(e^{\alpha \tau}-1\right)^{4}\right] \\
& +\frac{p(p-2)(p-4)}{48}\left[\left(e^{\alpha \tau}-1\right)^{6}+15\left(e^{\alpha \tau}-1\right)^{4} \hat{\sigma}^{2}\right. \\
& \left.+45\left(e^{\alpha \tau}-1\right)^{2} \hat{\sigma}^{4}+15 \hat{\sigma}^{6}\right]
\end{aligned}
$$

in which

$$
\hat{\sigma}=\sqrt{\frac{\sigma^{2}}{2 \alpha}\left(e^{2 \alpha \tau}-1\right)} .
$$

Proof. The proof is very technical so we divide it into 4 steps.

Step 1. We choose $p \in(0,1)$ for (2.6) to hold. Clearly, both $H_{1}(\tau)$ and $H_{2}(\tau)$ are continuous functions of $\tau$. It is easy to show that $H_{1}(0)=0$ and its derivative at $\tau=0$ is

$$
H_{1}^{\prime}(0)=p \alpha+\frac{p(p-1) \sigma^{2}}{2}=p\left(\alpha-\frac{(1-p) \sigma^{2}}{2}\right)<0,
$$

where the last inequality follows from condition (2.6). Moreover, by definition of $\hat{\sigma}$, we see that every term of $H_{2}(\tau)$ has a factor of either $\left(e^{\alpha \tau}-1\right)^{2}$ or $\left(e^{\alpha \tau}-1\right)\left(e^{2 \alpha \tau}-1\right)$ or $\left(e^{2 \alpha \tau}-1\right)^{2}$. Hence, $H_{2}(0)=0$ and $H_{2}^{\prime}(0)=0$. Consequently, $H_{1}(0)+H_{2}(0)=0$ and $H_{1}^{\prime}(0)+H_{2}^{\prime}(0)<0$. Therefore, if we let $\tau^{*}$ be the smallest positive root to the equation $H_{1}(\tau)+H_{2}(\tau)=0$, then

$$
H_{1}(\tau)+H_{2}(\tau)<0, \quad \forall \tau \in\left(0, \tau^{*}\right) .
$$

Step 2. From now on, let us now fix $\tau \in\left(0, \tau^{*}\right)$ arbitrarily. Let $t_{k}=k \tau$ for $k=0,1,2, \cdots$ and set $x_{k}=x\left(t_{k}\right)$. For 
$t \in\left[t_{k}, t_{k+1}\right], x(t)$ can be regarded as the solution to the following equation

$$
d x(t)=\alpha x(t) d t+\sigma x_{k} d B(t)
$$

with initial value $x\left(t_{k}\right)=x_{k}$ at time $t_{k}$. By the well-known variation-of-constants formula (see e.g. [18, Theorem 3.1 on page 96$]$ ), we have

$$
x(t)=x_{k} e^{\alpha\left(t-t_{k}\right)}+\int_{t_{k}}^{t} e^{\alpha(t-s)} \sigma x_{k} d B(s) .
$$

In particular,

$$
x_{k+1}=x_{k}\left(e^{\alpha \tau}+\sigma \xi_{k}\right),
$$

where $\xi_{k}=\int_{t_{k}}^{t_{k+1}} e^{\alpha\left(t_{k+1}-s\right)} d B(s)$. It is easy to see that $\xi_{k}$ is independent of $x_{k}$ and it has a normal distribution with mean 0 and variance

$$
\operatorname{Var}\left(\xi_{k}\right)=\int_{t_{k}}^{t_{k+1}} e^{2 \alpha\left(t_{k+1}-s\right)} d s=\frac{1}{2 \alpha}\left(e^{2 \alpha \tau}-1\right) .
$$

Hence, we can write $\xi_{k}=\sqrt{\frac{1}{2 \alpha}\left(e^{2 \alpha \tau}-1\right)} Z_{k}$, where $Z_{k} \sim$ $N(0,1)$ and is independent of $x_{k}$. It then follows from (2.14) that

$$
x_{k+1}=x_{k}\left(e^{\alpha \tau}+\hat{\sigma} Z_{k}\right) \text {, }
$$

where $\hat{\sigma}$ has been defined by (2.10) in the statement of the theorem. Consequently,

$$
\mathbb{E}\left|x_{k+1}\right|^{p}=\mathbb{E}\left|x_{k}\right|^{p} \mathbb{E}\left|e^{\alpha \tau}+\hat{\sigma} Z_{k}\right|^{p} .
$$

Rearrange

$$
\begin{aligned}
\left|e^{\alpha \tau}+\hat{\sigma} Z_{k}\right|^{p} & =\left(\left[1+e^{\alpha \tau}-1+\hat{\sigma} Z_{k}\right]^{2}\right)^{p / 2} \\
& =\left(1+2 Y_{k}+Y_{k}^{2}\right)^{p / 2}
\end{aligned}
$$

where $Y_{k}=e^{\alpha \tau}-1+\hat{\sigma} Z_{k}$. It is easy to see that $2 Y_{k}+Y_{k}^{2} \geq$ -1 . By the Taylor expansion theory, we can easily have the inequality

$$
\begin{aligned}
(1+u)^{p / 2} & \leq 1+\frac{p}{2} u+\frac{p(p-2)}{8} u^{2} \\
& +\frac{p(p-2)(p-4)}{48} u^{3}
\end{aligned}
$$

for $u \geq-1$. We then derive

$$
\begin{aligned}
& \left|e^{\alpha \tau}+\hat{\sigma} Z_{k}\right|^{p} \\
& \leq 1+\frac{p}{2}\left(2 Y_{k}+Y_{k}^{2}\right)+\frac{p(p-2)}{8}\left(2 Y_{k}+Y_{k}^{2}\right)^{2} \\
& +\frac{p(p-2)(p-4)}{48}\left(2 Y_{k}+Y_{k}^{2}\right)^{3} \\
& =1+p Y_{k}+\frac{p(p-1)}{2} Y_{k}^{2} \\
& +\frac{p(p-1)(p-2)}{6} Y_{k}^{3}+\frac{p(p-2)(2 p-7)}{8} Y_{k}^{4} \\
& +\frac{p(p-2)(p-4)}{8} Y_{k}^{5}+\frac{p(p-2)(p-4)}{48} Y_{k}^{6} .
\end{aligned}
$$

Making use of the properties of the standard normal distribution

$$
\mathbb{E}\left(Z_{k}^{2 n}\right)=(2 n-1) ! ! \quad \text { and } \quad \mathbb{E}\left(Z^{2 n-1}\right)=0
$$

for $n=1,2,3, \cdots$, where $(2 n-1) ! !=(2 n-1) \times(2 n-3) \times$ $\cdots \times 3 \times 1$, we can compute

$$
\left\{\begin{aligned}
\mathbb{E} Y_{k}= & e^{\alpha \tau}-1, \\
\mathbb{E} Y_{k}^{2}= & \left(e^{\alpha \tau}-1\right)^{2}+\hat{\sigma}^{2} \\
\mathbb{E} Y_{k}^{3}= & \left(e^{\alpha \tau}-1\right)^{3}+3\left(e^{\alpha \tau}-1\right) \hat{\sigma}^{2}, \\
\mathbb{E} Y_{k}^{4}= & \left(e^{\alpha \tau}-1\right)^{4}+6\left(e^{\alpha \tau}-1\right)^{2} \hat{\sigma}^{2}+3 \hat{\sigma}^{4}, \\
\mathbb{E} Y_{k}^{5}= & \left(e^{\alpha \tau}-1\right)^{5}+10\left(e^{\alpha \tau}-1\right)^{3} \hat{\sigma}^{2} \\
& +15\left(e^{\alpha \tau}-1\right) \hat{\sigma}^{4} \\
\mathbb{E} Y_{k}^{6}= & \left(e^{\alpha \tau}-1\right)^{6}+15\left(e^{\alpha \tau}-1\right)^{4} \hat{\sigma}^{2} \\
& +45\left(e^{\alpha \tau}-1\right)^{2} \hat{\sigma}^{4}+15 \hat{\sigma}^{6} .
\end{aligned}\right.
$$

We hence obtain from (2.19) that

$$
\mathbb{E}\left|e^{\alpha \tau}+\hat{\sigma} Z_{k}\right|^{p} \leq 1+H_{1}(\tau)+H_{2}(\tau),
$$

where $H_{1}(\tau)$ and $H_{2}(\tau)$ have been defined by (2.8) and (2.9), respectively, in the statement of the theorem.

Step 3. Recalling (2.11), we see that there is a unique $\varepsilon>0$ such that $1+H_{1}(\tau)+H_{2}(\tau)=e^{-\varepsilon \tau}$. It then follows from (2.16) and (2.21) that

$$
\mathbb{E}\left|x_{k+1}\right|^{p} \leq \mathbb{E}\left|x_{k}\right|^{p} e^{-\varepsilon \tau} .
$$

Since this holds for all $k \geq 0$, we get

$$
\mathbb{E}\left|x_{k+1}\right|^{p} \leq\left|x_{0}\right|^{p} e^{-\varepsilon(k+1) \tau}, \quad \forall k \geq 0 .
$$

Note from (2.13) that

$$
\begin{aligned}
& \mathbb{E}\left(\sup _{t_{k} \leq t \leq t_{k+1}}|x(t)|^{p}\right) \\
= & \mathbb{E}\left|x_{k}\right|^{p} \mathbb{E}\left(\sup _{t_{k} \leq t \leq t_{k+1}}\left|e^{\alpha\left(t-t_{k}\right)}+\sigma \int_{t_{k}}^{t} e^{\alpha(t-s)} d B(s)\right|^{p}\right) .
\end{aligned}
$$

But, by the elementary inequality $|a+b|^{p} \leq 2^{p}\left(|a|^{p}+|b|^{p}\right)$ for any real numbers $a$ and $b$, we derive

$$
\begin{aligned}
& \mathbb{E}\left(\sup _{t_{k} \leq t \leq t_{k+1}}\left|e^{\alpha\left(t-t_{k}\right)}+\sigma \int_{t_{k}}^{t} e^{\alpha(t-s)} d B(s)\right|^{p}\right) \\
\leq & 2^{p} \mathbb{E}\left(\sup _{t_{k} \leq t \leq t_{k+1}}\left[e^{p \alpha\left(t-t_{k}\right)}+|\sigma|^{p} e^{p \alpha t}\left|\int_{t_{k}}^{t} e^{-\alpha s} d B(s)\right|^{p}\right]\right) \\
\leq & 2^{p} e^{p \alpha \tau}+|2 \sigma|^{p} e^{p \alpha t_{k+1}} \mathbb{E}\left(\sup _{t_{k} \leq t \leq t_{k+1}}\left|\int_{t_{k}}^{t} e^{-\alpha s} d B(s)\right|^{p}\right) .
\end{aligned}
$$

Furthermore, by the Burkholder-Davis-Gundy inequality (see e.g. [15], [16]),

$$
\begin{aligned}
\mathbb{E}\left(\sup _{t_{k} \leq t \leq t_{k+1}}\left|\int_{t_{k}}^{t} e^{-\alpha s} d B(s)\right|^{p}\right) & \leq c_{p}\left(\int_{t_{k}}^{t_{k+1}} e^{-2 \alpha s} d s\right)^{p / 2} \\
& \leq c_{p} \tau^{p / 2} e^{-p \alpha t_{k}}
\end{aligned}
$$

where $c_{p}$ is a positive number dependent on $p$ only. We therefore obtain that

$$
\mathbb{E}\left(\sup _{t_{k} \leq t \leq t_{k+1}}\left|e^{\alpha\left(t-t_{k}\right)}+\sigma \int_{t_{k}}^{t} e^{\alpha(t-s)} d B(s)\right|^{p}\right) \leq C,
$$

where $C=2^{p} e^{p \alpha \tau}\left(1+c_{p}|\sigma|^{p} \tau^{p / 2}\right)$ independent of $k$. Substituting this into (2.23) and then making use of (2.22), we have

$$
\mathbb{E}\left(\sup _{t_{k} \leq t \leq t_{k+1}}|x(t)|^{p}\right) \leq C\left|x_{0}\right|^{p} e^{-\varepsilon k \tau}, \quad \forall k \geq 0 .
$$


Step 4. It follows from (2.24) that

$$
\begin{aligned}
\mathbb{P}\left(\sup _{t_{k} \leq t \leq t_{k+1}}|x(t)|^{p} \geq e^{-0.5 \varepsilon k \tau}\right) & \leq \frac{\mathbb{E}\left(\sup _{t_{k} \leq t \leq t_{k+1}}|x(t)|^{p}\right)}{e^{-0.5 \varepsilon k \tau}} \\
& \leq C\left|x_{0}\right|^{p} e^{-0.5 \varepsilon k \tau}, \quad \forall k \geq 0 .
\end{aligned}
$$

By the well-known Borel-Cantelli lemma (see e.g. [18, Lemma 2.4 on page 7 ]), we see that

$$
\sup _{t_{k} \leq t \leq t_{k+1}}|x(t)|^{p}<e^{-0.5 \varepsilon k \tau}
$$

holds for all but finitely many $k$. That is, for almost all $\omega \in \Omega$, there is an integer $k_{0}=k_{0}(\omega)$ such that

$$
\sup _{t_{k} \leq t \leq t_{k+1}}|x(t, \omega)|^{p}<e^{-0.5 \varepsilon k \tau} \quad \forall k \geq k_{0}(\omega) .
$$

Therefore, for $t_{k} \leq t \leq t_{k+1}$ and $k \geq k_{0}$,

$$
\frac{1}{t} \log (|x(t, \omega)|)<-\frac{0.5 \varepsilon k \tau}{p(k+1) \tau} \text {. }
$$

Letting $t \rightarrow \infty$, we get

$$
\limsup _{t \rightarrow \infty} \frac{1}{t} \log (|x(t, \omega)|) \leq-\frac{\varepsilon}{2 p}
$$

for almost all $\omega \in \Omega$. The proof is hence complete.

\section{Multi-Dimensional Nonlinear Equations}

Let us now generalize our theory in the previous section to the multi-dimensional nonlinear case. Consider a nonlinear $n$-dimensional unstable ODE $\frac{d y(t)}{d t}=f(y(t))$ and its corresponding stochastically controlled system

$$
d x(t)=f(x(t)) d t+A x\left(\delta_{t}\right) d B(t), \quad t \geq 0,
$$

where $f: \mathbb{R}^{n} \rightarrow \mathbb{R}^{n}, A \in \mathbb{R}^{n \times n}$ and $\delta_{t}=[t / \tau] \tau$. We impose the following assumptions.

Assumption 3.1: Assume that $f$ is globally Lipschitz continuous, namely

$$
|f(x)-f(y)| \leq \alpha|x-y|, \quad x, y \in \mathbb{R}^{n},
$$

where $\alpha>0$. For the stability purpose of this paper, we also assume that $f(0)=0$.

Assumption 3.2: There are two positive constants $\rho_{1}$ and $\rho_{2}$ such that

$$
\rho_{2}-0.5 \rho_{1}>\alpha
$$

and, for all $x \in \mathbb{R}^{n}$,

$$
|A x|^{2} \leq \rho_{1}|x|^{2} \quad \text { and } \quad\left|x^{T} A x\right|^{2} \geq \rho_{2}|x|^{4} .
$$

Note that Assumption 3.1 implies the linear growth condition

$$
|f(x)| \leq \alpha|x|, \quad x \in \mathbb{R}^{n} .
$$

It is known (see, e.g., [18]) that there are many examples of the square matrix $A$ that fulfils Assumption 3.2.

Theorem 3.3: Let Assumptions 3.1 and 3.2 hold. Then there is a positive number $\tau^{*}$ such that for any initial value $x_{0} \in \mathbb{R}$, the solution of equation (3.1) satisfies

$$
\limsup _{t \rightarrow \infty} \frac{1}{t} \log (|x(t)|)<0 \quad \text { a.s. }
$$

provided $\tau \in\left(0, \tau^{*}\right)$. In practice, we can choose a pair of constants $p, \varepsilon \in(0,1)$ for $(3.8)$ to hold and let $\tau^{*}=\bar{\tau}$, where $\bar{\tau}>0$ is the unique root to equation (3.13) defined below.

To prove the theorem, we present a couple of lemmas. We denote by $x\left(t ; x_{0}\right)$ the solution of equation (3.1) with initial value $x(0)=x_{0} \in \mathbb{R}^{n}$ and by $y\left(t ; y_{0}\right)$ the solution of the following $\mathrm{SDE}$

$$
d y(t)=f(y(t)) d t+A y(t) d B(t)
$$

on $t \geq 0$ with initial value $y(0)=y_{0} \in \mathbb{R}^{n}$.

Lemma 3.4: Let Assumption 3.1 and 3.2 hold. Choose $p \in$ $(0,1)$ sufficiently small for which

$$
(1-0.5 p) \rho_{2}-0.5 \rho_{1}>\alpha .
$$

Then, for all $y_{0} \in \mathbb{R}^{n}$,

$$
\mathbb{E}\left|y\left(t ; y_{0}\right)\right|^{p} \leq\left|y_{0}\right|^{p} e^{-\gamma t}, \quad \forall t \geq 0,
$$

where $\gamma=p\left[(1-0.5 p) \rho_{2}-0.5 \rho_{1}-\alpha\right]$.

Proof. We only need to prove the assertion for $y_{0} \neq 0$. For any $y_{0} \neq 0$, it is known (see [18, Lemma 3.2 on p120]) that $y\left(t ; y_{0}\right) \neq 0$ for all $t \geq 0$ almost surely. Write $y\left(t ; y_{0}\right)=y(t)$. By the Itô formula and assumptions 3.1 and 3.2, we can derive that

$$
\begin{aligned}
d\left(|y(t)|^{p}\right) & =\left(0.5 p|y(t)|^{p-2}\left[2 y^{T}(t) f(y(t))+|A y(t)|^{2}\right]\right. \\
& \left.-(1-0.5 p)|y(t)|^{p-4}\left|y^{T}(t) A y(t)\right|^{2}\right) d t \\
& +p|y(t)|^{p-2} y^{T}(t) A y(t) d B(t) \\
& \leq-\gamma|y(t)|^{2} d t+p|y(t)|^{p-2} y^{T}(t) A y(t) d B(t)
\end{aligned}
$$

This implies easily that

$$
\mathbb{E}\left(e^{\gamma t}|y(t)|^{p}\right) \leq\left|y_{0}\right|^{p}
$$

and hence the assertion follows. The proof is complete.

Lemma 3.5: Let Assumptions 3.1 and 3.2 hold. Then

$$
\begin{aligned}
& \mathbb{E}\left|y\left(t ; x_{0}\right)-x\left(t ; x_{0}\right)\right|^{p} \\
& \leq\left|x_{0}\right|^{p} K(\tau, p)\left[e^{\left.\left(4 \alpha+3\|A\|^{2}\right)\right) t}-1\right]^{p / 2}
\end{aligned}
$$

for all $x_{0} \in \mathbb{R}$ and $t \geq 0$, where

$$
K(\tau, p)=\left(\frac{4 \tau\|A\|^{2}\left(\alpha^{2} \tau+\|A\|^{2}\right)}{2 \alpha+\|A\|^{2}}\right)^{p / 2} .
$$

Proof. Fix any $x_{0} \in \mathbb{R}$ and write $x\left(t ; x_{0}\right)=x(t)$ and $y\left(t ; x_{0}\right)=y(t)$. By the Itô formula and Assumption 3.1, it is easy to show

$$
\begin{aligned}
& \mathbb{E}|x(t)-y(t)|^{2} \\
\leq & 2\left(\alpha+\|A\|^{2}\right) \int_{0}^{t} \mathbb{E}|x(s)-y(s)|^{2} d s \\
+ & \left.2\|A\|^{2} \int_{0}^{t} \mathbb{E}\left|x(s)-x\left(\delta_{s}\right)\right|^{2}\right] d s .
\end{aligned}
$$

The Gronwall inequality then implies

$$
\mathbb{E}|y(t)-x(t)|^{2} \leq 2 \sigma^{2} e^{2 t\left(\alpha+\|A\|^{2}\right)} \int_{0}^{t} \mathbb{E}\left|x(s)-x\left(\delta_{s}\right)\right|^{2} d s .
$$


On the other hand, it is very easy to show, by the Itô formula, that

$$
\mathbb{E}|x(t)|^{2} \leq\left|x_{0}\right|^{2} e^{\left(2 \alpha+\|A\|^{2}\right) t}
$$

and then

$$
\mathbb{E}\left|x(t)-x\left(\delta_{t}\right)\right|^{2} \leq 2 \tau\left(\alpha^{2} \tau+\|A\|^{2}\right)\left|x_{0}\right|^{2} e^{\left(2 \alpha+\|A\|^{2}\right) t} .
$$

Substituting this into (3.11) yields

$$
\begin{aligned}
& \mathbb{E}|y(t)-x(t)|^{2} \\
& \leq \frac{4 \tau\|A\|^{2}\left(\alpha^{2} \tau+\|A\|^{2}\right)}{2 \alpha+\|A\|^{2}}\left|x_{0}\right|^{2}\left[e^{\left.\left(4 \alpha+3\|A\|^{2}\right)\right) t}-1\right] .
\end{aligned}
$$

A simple application of the Hölder inequality implies the desired assertion (3.10). The proof is complete.

Lemma 3.6: Let Assumption 3.1 and 3.2 hold. Choose a pair of constants $p, \varepsilon \in(0,1)$ for (3.8) to hold. Let $\bar{\tau}>0$ be the unique root to the equation

$$
2^{p} K(\tau, p)\left[e^{\left(4 \alpha+3\|A\|^{2}\right)\left[\tau+\log \left(2^{p} / \varepsilon\right) / \gamma\right]}-1\right]^{p / 2}=1-\varepsilon,
$$

where $\gamma$ and $K(\tau, p)$ have been defined in Lemmas 3.4 and 3.5. Then, for each $\tau \in(0, \bar{\tau})$, there is a pair of positive numbers $\bar{k}$ and $\lambda$ such that, for all initial value $x_{0} \in \mathbb{R}^{n}$, the solution of equation (3.1) satisfies

$$
\mathbb{E}\left|x\left(i \bar{k} \tau ; x_{0}\right)\right|^{p} \leq\left|x_{0}\right|^{p} e^{-\lambda i \bar{k} \tau}, \quad \forall i=1,2, \cdots .
$$

Proof. We first observe that when $p$ is fixed, the righthand-side term of equation (3.13) is a continuously increasing function of $\tau \geq 0$ and equals to zero when $\tau=0$ so equation (3.13) must have a unique root $\bar{\tau}>0$. Fix $\tau \in(0, \bar{\tau})$ and $x_{0} \in \mathbb{R}$ arbitrarily and write $x\left(i \tau ; x_{0}\right)=x_{i}$ for $i=0,1,2, \cdots$. Choose a positive integer $\bar{k}$ such that

$$
\frac{\log \left(2^{p} / \varepsilon\right)}{\gamma \tau} \leq \bar{k}<1+\frac{\log \left(2^{p} / \varepsilon\right)}{\gamma \tau},
$$

where $\gamma$ has been defined in Lemma 3.4. So

$$
2^{p} e^{-\gamma \bar{k} \tau} \leq \varepsilon
$$

Write $y\left(\bar{k} \tau ; x_{0}\right)=y_{\bar{k}}$. By Lemma 3.4,

$$
\mathbb{E}\left|y_{\bar{k}}\right|^{p} \leq\left|x_{0}\right|^{p} e^{-\gamma \bar{k} \tau} .
$$

By the elementary inequality $(a+b)^{p} \leq 2^{p}\left(a^{p}+b^{p}\right)$ for any $a, b \geq 0$, we have

$$
\mathbb{E}\left|x_{\bar{k}}\right|^{p} \leq 2^{p} \mathbb{E}\left|y_{\bar{k}}\right|^{p}+2^{p} \mathbb{E}\left|y_{\bar{k}}-x_{\bar{k}}\right|^{p} .
$$

Using (3.17) along with (3.16) as well as Lemma 3.5, we get

$$
\mathbb{E}\left|x_{\bar{k}}\right|^{p} \leq\left|x_{0}\right|^{p}\left(\varepsilon+2^{p} K(\tau, p)\left[e^{\left(4 \alpha+3\|A\|^{2}\right) \bar{k} \tau}-1\right]^{p / 2}\right) .
$$

But, by (3.15),

$$
e^{\left(4 \alpha+3\|A\|^{2}\right) \bar{k} \tau} \leq e^{\left(4 \alpha+3\|A\|^{2}\right)\left[\tau+\log \left(2^{p} / \varepsilon\right) / \gamma\right]} .
$$

We hence see from (3.13) that

$$
\begin{aligned}
& \varepsilon+2^{p} K(\tau, p)\left[e^{\left(4 \alpha+3\|A\|^{2}\right) \bar{k} \tau}-1\right]^{p / 2} \\
\leq & \varepsilon+2^{p} K(\tau, p)\left[e^{\left(4 \alpha+3\|A\|^{2}\right)\left[\tau+\log \left(2^{p} / \varepsilon\right) / \gamma\right]}-1\right]^{p / 2}
\end{aligned}
$$$$
<1 \text {. }
$$

We may therefore write

$$
\varepsilon+2^{p} K(\tau, p)\left[e^{\left(4 \alpha+3\|A\|^{2}\right) \bar{k} \tau}-1\right]^{p / 2}=e^{-\lambda \bar{k} \tau}
$$

for some $\lambda>0$. It then follows from (3.18) that

$$
\mathbb{E}\left|x_{\bar{k}}\right|^{p} \leq\left|x_{0}\right|^{p} e^{-\lambda \bar{k} \tau} .
$$

Let us now consider the solution $x(t)$ of equation (3.1) on $t \geq \bar{k} \tau$. This can be regarded as the solution of equation (3.1) starting from $x_{\bar{k}}$ at $t=\bar{k} \tau$. Due to the time-homogeneous property of equation (3.1), we therefore see easily that

$$
\mathbb{E}\left(\left|x_{2 \bar{k}}\right|^{p} \mid \mathcal{F}_{\bar{k} \tau}\right) \leq\left|x_{\bar{k}}\right|^{p} e^{-\lambda \bar{k} \tau} .
$$

This implies

$$
\mathbb{E}\left|x_{2 \bar{k}}\right|^{p} \leq \mathbb{E}\left|x_{\bar{k}}\right|^{p} e^{-\lambda \bar{k} \tau} \leq\left|x_{0}\right|^{p} e^{-2 \lambda \bar{k} \tau} .
$$

Repeating this procedure, we have

$\mathbb{E}\left|x_{i \bar{k}}\right|^{p} \leq \mathbb{E}\left|x_{(i-1) \bar{k}}\right|^{p} e^{-\lambda \bar{k} \tau} \leq\left|x_{0}\right|^{p} e^{-\lambda i \bar{k} \tau}, \quad \forall i=1,2, \cdots$,

as desired. The proof is hence complete.

Proof of Theorem 3.3. Choose a pair of positive constants $p, \varepsilon \in(0,1)$ for $(3.8)$ to hold and let $\tau^{*}=\bar{\tau}$, where $\bar{\tau}$ is the unique root to equation (3.13). Fix $\tau \in\left(0, \tau^{*}\right)$ and $x_{0} \in \mathbb{R}$ arbitrarily and write $x\left(t ; x_{0}\right)=x(t)$. Also let $\bar{k}$ be the same as in Lemma 3.6 and $x_{i \bar{k}}$ as defined in the proof of Lemma 3.6. For $t \in[0, \bar{k} \tau]$, it follows from equation (3.1) that

$$
\left.x(t)=x_{0}+\int_{0}^{t} f(x(s)) d s+\int_{0}^{t} A x\left(\delta_{s}\right)\right) d B(s) .
$$

By the Hölder inequality and the Burkholder-Davis-Gandy inequality and condition (3.5), we derive that, for $0 \leq u \leq \bar{k} \tau$,

$$
\begin{aligned}
& \mathbb{E}\left(\sup _{0 \leq t \leq u}|x(t)|^{2}\right) \\
\leq & 3\left|x_{0}\right|^{2}+3 \mathbb{E}\left(\left[\int_{0}^{t} \alpha|x(s)| d s\right]^{2}\right) \\
+ & 3 \mathbb{E}\left(\sup _{0 \leq t \leq u}\left|\int_{0}^{u} A x\left(\delta_{s}\right) d B(s)\right|^{2}\right) \\
\leq & 3\left|x_{0}\right|^{2}+3\left(\alpha^{2} \bar{k} \tau+4\|A\|^{2}\right) \int_{0}^{u} \mathbb{E}\left(\sup _{0 \leq t \leq s}|x(t)|^{2}\right) d s .
\end{aligned}
$$

The Gronwall inequality shows that

$$
\mathbb{E}\left(\sup _{0 \leq t \leq \bar{k} \tau}|x(t)|^{2}\right) \leq 3\left|x_{0}\right|^{2} e^{3 \bar{k} \tau\left(\alpha^{2} \bar{k} \tau+4\|A\|^{2}\right)} .
$$

The Hölder inequality then gives

$$
\mathbb{E}\left(\sup _{0 \leq t \leq \bar{k} \tau}|x(t)|^{p}\right) \leq K\left|x_{0}\right|^{p}
$$

where $K=3^{p / 2} e^{1.5 p \bar{k} \tau\left(\alpha^{2} \bar{k} \tau+4\|A\|^{2}\right)}$.

Let us now consider the solution $x(t)$ of equation (3.1) on $t \in[i \bar{k} \tau,(i+1) \bar{k} \tau]$ for $i=1,2, \cdots$. This can be regarded as the solution of equation (3.1) starting from $x_{i \bar{k}}$ at $t=i \bar{k} \tau$. Due to the time-homogeneous property of equation (3.1), we therefore see easily from (3.21) that

$$
\mathbb{E}\left(\sup _{i \bar{k} \tau \leq t \leq(i+1) \bar{k} \tau}|x(t)|^{p} \mid \mathcal{F}_{i \bar{k} \tau}\right) \leq K\left|x_{i \bar{k}}\right|^{p} .
$$


This, together with Lemma 3.6, implies

$$
\mathbb{E}\left(\sup _{i \bar{k} \tau \leq t \leq(i+1) \bar{k} \tau}|x(t)|^{p}\right) \leq K \mathbb{E}\left|x_{i \bar{k}}\right|^{p} \leq K\left|x_{0}\right|^{p} e^{-\lambda i \bar{k} \tau}
$$

for all $i \geq 1$. Consequently

$$
\mathbb{P}\left(\sup _{i \bar{k} \tau \leq t \leq(i+1) \bar{k} \tau}|x(t)|^{p} \geq e^{-0.5 \lambda i \bar{k} \tau}\right) \leq K\left|x_{0}\right|^{p} e^{-0.5 \lambda i \bar{k} \tau}
$$

for all $i \geq 1$. The well-known Borel-Cantelli lemma yields

$$
\sup _{i \bar{k} \tau \leq t \leq(i+1) \bar{k} \tau}|x(t)|^{p}<e^{-0.5 \lambda i \bar{k} \tau}
$$

holds for all but finitely many $i$. That is, for almost all $\omega \in \Omega$, there is an integer $i_{0}=i_{0}(\omega)$ such that

$$
\sup _{i \bar{k} \tau \leq t \leq(i+1) \bar{k} \tau}|x(t, \omega)|^{p}<e^{-0.5 \lambda i \bar{k} \tau} \quad \forall i \geq i_{0}(\omega) .
$$

Therefore, for $i \bar{k} \tau \leq t \leq(i+1) \bar{k} \tau$ and $i \geq i_{0}$,

$$
\frac{1}{t} \log (|x(t, \omega)|)<-\frac{0.5 \lambda i \bar{k} \tau}{p(i+1) \bar{k} \tau} \text {. }
$$

Letting $t \rightarrow \infty$, we get

$$
\limsup _{t \rightarrow \infty} \frac{1}{t} \log (|x(t, \omega)|) \leq-\frac{\lambda}{2 p}
$$

for almost all $\omega \in \Omega$. The proof is hence complete.

\section{Conclusions}

It is well known that noise can be used to stabilize a given unstable system or to make a system even more stable when it is already stable. However, all results in this area so far require the stochastic feedback control depend on the current state $x(t)$ continuously. In this paper we initiate the study of stabilization by a stochastic feedback control dependent on only discretetime states $x(k \tau)$. We discuss the scalar linear case as well as the multi-dimensional nonlinear case. Our theory shows clearly that it is possible to stabilize a given unstable system by a discrete-time stochastic feedback control. In this paper, we only use a scalar Brownian motion to avoid the notation becoming too complicated but our theory works for multidimensional Brownian motions and we leave the details to the reader. We should piont out that we do not know how to determine the optimal value for $\tau^{*}$, even in the linear case. We have a feeling that it is very hard to determine the optimal value. However, it is very useful in practice even if we could improve the bound for $\tau^{*}$.

\section{ACKNOWLEDGEMENTS}

The author would like to thank the associate editor and the referees for their very helpful comments and suggestions. He would also like to thank the Leverhulme Trust (RF -2015-385), the EPSRC (EP/E009409/1), the Royal Society of London (IE131408), the Royal Society of Edinburgh (RKES115071), the London Mathematical Society (11219), the Edinburgh Mathematical Society (RKES130172), the State Administration of Foreign Experts Affairs of China (MS2014DHDX020) and the Ministry of Education (MOE) of China for their financial support.

\section{REFERENCES}

[1] Allwright, J.C., Astolfi, A. and Wong, H.P., A note on asymptotic stabilization of linear systems by periodic, piecewise constant, output feedback, Automatica 41(2) (2005), 339-344.

[2] Appleby, J. and Mao, X., Stochastic stabilization of functional differential equations, Systems and Control Letters 54(11) (2005), 1069-1081.

[3] Arnold, L., Stochastic Differential Equations: Theory and Applications, John Wiley and Sons, 1974.

[4] Arnold, L., Crauel, H. and Wihstutz, V., Stabilization of linear systems by noise, SIAM J. Control Optim. 21 (1983), 451-461.

[5] Black, F. and Scholes, M., The prices of options and corporate liabilities, J. Political Economy 81 (1973), 637-654.

[6] Ebihara, Y., Yamaguchi, J. and Hagiwara, T., Periodically time-varying controller synthesis for multiobjective $\mathrm{H}_{2} / \mathrm{H}_{\infty}$ control of discrete-time systems and analysis of achievable performance, Systems Control Lett. 60(9) (2011), 709-717.

[7] Chammas, A.B. and Leondes, C.T., On the finite time control of linear systems by piecewise constant output feedback, Internat. J. Control 30(2) (1979), 227-234.

[8] Chen,T.W. and Francis, B., Optimal Sampled Data Control Systems, Springer-Verlag, London, 1995.

[9] Fernholz, E.R. and Karatzas, I., Relative arbitrage in volatility-stailized markets, Ann. Finance 1 (2005), 149-177.

[10] Hagiwara,T. and Araki, M., Design of stable state feedback controller based on the multirate sampling of the plant output, IEEE Trans. Automat. Control 33(9) (1988), 812-819.

[11] Hagiwara,T. and Araki, M., On preservation of strong stabilizability under sampling. IEEE Trans. Automat. Control 33(11) (1988), 10801082.

[12] Has'minskii, R.Z., Stochastic Stability of Differential Equations, Sijthoff and Noordhoff, 1981.

[13] Kolmanovskii, V.B. and Myshkis, A., Applied Theory of Functional Differential Equations, Kluwer Academic Publishers, 1992.

[14] Ladde, G.S. and Lakshmikantham, V., Random Differential Inequalities, Academic Press, 1980.

[15] Mao, X., Stability of Stochastic Differential Equations with Respect to Semimartingales, Longman Scientific and Technical, 1991.

[16] Mao, X., Exponential Stability of Stochastic Differential Equations, Marcel Dekker, 1994.

[17] Mao, X., Stochastic stabilisation and destabilisation, Systems and Control Letters 23 (1994), 279-290.

[18] Mao X., Stochastic Differential Equations and Their Applications, 2nd Edition, Horwood Pub., 2007.

[19] Mao, X., Stability and stabilization of stochastic differential delay equations, IET Control Theory \& Applications 1(6) (2007), 1551-1566.

[20] Mao, X., Lam, J. and Huang, L., Stabilisation of hybrid stochastic differential equations by delay feedback control, Systems \& Control Letters 57 (2008), 927-935.

[21] Mao, X. and Yuan, C., Stochastic Differential Equations with Markovian Switching, Imperial College Press, 2006.

[22] Mao, X., Yin, G. and Yuan, C., Stabilization and destabilization of hybrid systems of stochastic differential equations, Automatica 43 (2007), 264273.

[23] Mohammed, S.-E.A., Stochastic Functional Differential Equations, Longman Scientific and Technical, 1986.

[24] Mohammed, S.-E.A. and Scheutzow, M.K.R., Lyapunov exponents of linear stochastic functional differential equations driven by semimartingales: Part I. The multiplicative ergodic theory, Ann. Inst. H. Poincare Probab. Statist. 32 (1996), 69-105.

[25] Pinsky, M.A. and Wihstutz, V., Lyapunov exponents of nilponent Itô systems, Stochastics 25 (1988), 43-57.

[26] Scheutzow, M.K.R., Stabilisation and destabilisation by noise in the plane, Stoch. Anal. Appl. 11(1) (1993), 97-113. 